\title{
Innovative PCG technique for Cardiac Spectral Analysis
}

\author{
Prof. D. P. Sharma \\ Dept. of Computer Science, ASBM, India
}

\begin{abstract}
Normally the ratio of population versus physician is so feeble in most of the economically underdeveloped and developing countries that cause delayed diagnosis and non-availability of on-time treatment of heart patients specifically in the rural areas. It forces to think about availability of a low cost equipment in the rural health centres which may readily diagnose and alarm the critical cardiac condition of patient in absence of cardiologist too, so that the patient can immediately be carried to nearby urban health centre for early treatment.

This paper presents an effective and handy PCG device which is equally useful for urban health centres to assist the cardiologist in making faster decision and control of the ailment, which is vital and critical subject in treating such patients.

The equipment developed is low cost, quiet affordable by anyone or the rural health centres, portable and easy to maneuver by anyone who possess even a little knowledge to handle it and read the analysis. It does not require any specialized medical person to use the equipment. The diagnosis is carried through computerbased inference system which counts for reliability and accuracy of the diagnosis.
\end{abstract}

Keywords - Phonocardiograph, PCG device, Human Heart, Osculation, Spectral Analysis,

\section{Introduction}

With the advent of the stethoscope in the year 1816, the technique of heart auscultation entered the domain of modern medical analysis. Prior to the modern practice, a remarkable number of fresh medical practicener fail to diagnose heart sounds by just listening to it due to the restriction of the human ear to detect low frequency heart sounds, and therefore the proper diagnosis of the heart was not possible. This paved the way for Phonocardiogram equipment, where study of the heart sound waveform enabled professionals to determine the nature of heart disease.

The Phonocardiogram (PCG) device is quite a well-known device, which is used to listen to the heart sounds of a human being and the output from the machine is compared with the ideal heart sounds of a human being of that age and the deviation from the ideal response is then analyzed to detect the nature of ailment.

The PCG devices in use today are very expensive and usually detect three peaks of the heart sounds. While the PCG device described here is such that observation and analysis of the waveforms can be done with ease. Any trained personnel, which always may not be the doctor himself, can operate this device and analyze its waveforms. This device is extremely cost effective; doctors can use it in their clinics too.

Along with a cost effective alternative to the expensive options, it is sufficiently sensitive also to catch even a minute change in the heart sound.

It uses computer to display and analyze the waveform which is nowadays easily available everywhere.

\section{The Human Heart}

The heart is a muscular organ responsible for pumping blood through the blood vessels by repeated, rhythmic contractions. The heart is composed of the cardiac muscle, an involuntary muscle tissue which is found only within this organ. The cardiac valves maintain unidirectional flow of blood by opening and closing, depending on the difference in pressure on each side.

There are four valves of the heart (not counting the valve of the coronary sinus and valve of the inferior vena cava). The two atrioventricular (AV) valves ensure that blood flows from the atria to the ventricles, and not the other way. The two semilunar (SL) valves are present in the arteries leaving the heart, and they prevent the blood flowing back from the arteries into the ventricles. The sound of heart valves shutting causes the heart sounds.

Heart's sounds are generated within the heart during cardiac cycle. The main cause of these sounds is acceleration and deceleration of blood flow, and closing and opening of the heart valves. Normally there are two heart sounds, and any additional sounds indicate diseased condition of cardiac. If a third heart sound is present it could be a sign of cardiac failure whereas a murmur indicates defective valves or an orifice in the septal wall. There are four sounds produced in the heart, namely: 
First Heart Sound: It is heard at the onset of the ventricular systole, during the closure of the AV valves. It is of longer duration than the other heart sounds and the frequency is around 25 to $45 \mathrm{~Hz}$. It is called "Lub". The causes of the first heart sound are mainly due to three reasons: Vascular, Muscular and Valvular.

- Vascular cause is due to the turbulence created in the blood due to ventricular contraction in it.

- Muscular cause is due to the vibration of the walls of the contracting ventricles.

- Valvular cause is due to the vibration of the walls of the valve cusps during closure.

Second Heart Sound: It is heard at the end of clinical systole during closure of the semi lunar valves. It is of shorter duration and is of higher frequency $(50 \mathrm{~Hz})$ than the first heart sound. It is called "Dub". The causes of the second heart sound are mainly also due to the three reasons: Vascular, Muscular and Valvular.

- Vascular cause is due to the oscillation/vibration of the blood column in the aorta and in the pulmonary trunk.

- Muscular cause here means vibration of the walls of the pulmonary trunk and the ascending aorta.

- Valvular cause is due to the closure of the semi lunar valves.

Third Heart Sound: It is a low pitched soft sound of 0.1 second duration, heard in the early diastole and is produced due to the first rapid filling phase, i.e., after the second sound. It occurs probably due to vibration in the ventricular wall, caused by the movement of blood. It can be heard sometimes in normal children and young individuals with thin chest and high venous return. Third sound in abnormal situation signifies heart failure.

Fourth Heart Sound: It is also called arterial sound and is produced during arterial contraction. It is heard just before the first sound i.e. late in the diastole. It is believed to be produced when the atria are forcefully trying to pump blood into noncompliant ventricles. It is not heard in case of a normal individual. In abnormal situations like heart failure, after myocardial infarctions, the fourth sound is often heard.

\section{Methodology}

Phonocardiograph signals contain bio-acoustic information reflecting operation of the heart. The primary objective of this paper is to use computer based signal processing to improve the diagnostic value of this information.

- A nonlinear change detection method has been applied to automatically detect heart sounds. The first and the second heart sounds can be found using recurrence times of the first kind while the third heart sound can be found using recurrence times of the second kind. Most of the third heart sound occurrences were detected $(98 \%)$, but the amount of false extra detections was rather high (7\% of the heart cycles).

- Spectral analysis of waveform obtained from patient's osculation is carried along with its sampling, and comparing with cardiac database to diagnose the present condition of patient.

The method discussed gives reasonable results, and it provide a step forward in the quest for an intelligent osculation by improving sound quality, emphasizing abnormal events in the heart cycle and distinguishing different heart sounds.

\section{The Phonocardiogram (PCG) Equipment}

In this paper, the attempt has been taken to design a PCG machine with very basic electronic components that could detect two of the peaks of the cardiac sounds efficiently and often give the third and fourth peak too, as also an audio output and an oscilloscope and PC output. The manufacturing cost of the device is also surprisingly less, thereby making it an affordable instrument with considerable efficiency. See Figure 1 for schematic block diagram of the proposed equipment. 


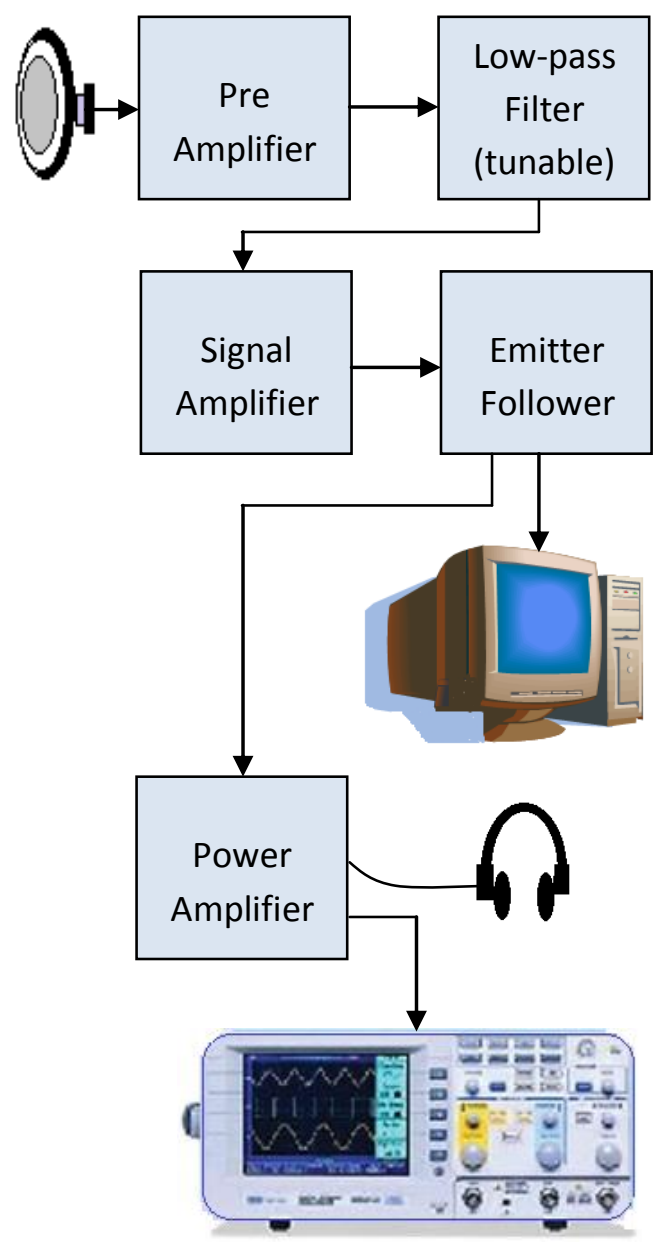

Figure 1: Block diagram of the proposed PCG machine

A small microphone embedded inside the stethoscope's ear pipe is used to capture sound impulses produced by the heart valves that pass through stethoscope diaphragm. It works as a transducer and generates an output electrical signal, which is in the range of a few micro volts.

Since output of the microphone is very weak enough, thus this signal needs to be amplified and made free from noise, before it can be processed further and displayed on cathode ray oscilloscope (CRO) and PC. It has been observed that the output of the microphone need an amplification at around 5000 to get a signal of suitable magnitude of voltage to be displayed on cathode ray oscilloscope. This amplification is carried in two stages. In the first (signal frequency amplification) stage, an Op-Amp is used as amplifier. IC-741 can be one of the best choices for this purpose. At this stage an inverting mode of operation is used and the gain is kept to around 50. The signal voltage, after amplification of 50 gives an output in millivolts range. The signal is now amplified.

Next, the noise present in the system needs to be reduced. This is done by passing the output signal from the above circuit through a Low-Pass Filter having a cut-off frequency of approximately $200 \mathrm{~Hz}$.

Cardiac signals are known to have a frequency of around $20 \mathrm{~Hz}$ to $200 \mathrm{~Hz}$, with an average of around $140 \mathrm{~Hz}$. Hence, a first order low pass filter having a cut off frequency of $200 \mathrm{~Hz}$ is to be designed. This ensures that the high frequency noise components will be blocked and that they will not interfere any further with the main signal.

This is advisable to keep the filter is a tunable one to ensure that the individual heart sounds can be analyzed to obtain maximum efficiency. The output from the filter is now free from noise. We can opt for OpAmp IC-741 again as one of the best options.

This output of filter is now to be fed to the Signal frequency (Common-Emitter) Amplifier, where it is amplified once more. As mentioned earlier, the total amplification required in the system will be around 5000. A Common-Emitter Amplifier is used to achieve the remaining amplification. Had the total amplification been 
given before the filtering stage, the noise signals would also have been amplified, which would have led to further trouble! Also, a common emitter amplifier is to be designed with a gain of 100, which will have an adequate frequency response. Increasing the gain by changing the design parameters may have an adverse effect as the frequency response becomes poor. So a basic amplifier should be chosen with a gain of 50 (5000/100) and a C-E amplifier with a gain of 100. As these two are cascaded, the net gain of 5000 can be easily achieved.

The output from the CE Amplifier is now fed to the input of an Emitter Follower circuit. This is done for the purpose of impedance matching. The output from this stage is also fed into the sound card of a computer, through the AUDIO IN Port.

The output impedance of a common emitter amplifier might be quite high. Now, apart from displaying the signal on a CRO, an audio output, whose impedance must be low, will also be required. Hence, the problem of impedance mismatch might occur. To avoid this problem, an emitter follower circuit is to be used. An emitter follower is a circuit whose output closely follows the input; however, there is a difference between the impedance of the input and that of the output.

The emitter follower that we use will have high input impedance and low output impedance. Hence, this circuit basically acts as a buffer stage that performs the function of impedance transformation over a wide range of frequencies with voltage gain close to unity. In addition, the emitter follower also increases the power level of the signal which is of great help to us, as the output of the emitter follower is to be fed into a bridge amplifier. It should also be noted that in the emitter follower circuit that we use, there is no phase shift between the output and input, in either voltage or current.

The signal is to be finally fed to the power amplification stage. Here the signal obtained from the Emitter-Follower is processed, so as to be able to feed it to a sub-woofer system. IC: TDA 2004 can be one of the choices for this purpose. It is a dual $9 \mathrm{~W}$ amplifier that is housed in an 11-pin package. The chip can operate from single-ended supplies in the range of 8 to $18 \mathrm{~V}$, can provide peak output currents of $3.5 \mathrm{~A}$ and can deliver $9 \mathrm{~W}$ into a 4R0 load from each channel, using a 17V supply.

Since the Digital Oscilloscope is normally not available. In order to make the device more user friendly, it was necessary to display the heart sound waveform in a Personal Computer or a Laptop that is readily available to a greater percentage of the population. For viewing the waveforms, open source software by the name of Audacity ver. 1.2.6 is used and it is available free of cost at www.audacity.sourceforge.net.

\section{Observations}

The performance of the system as a whole is now being considered and a critical analysis of this project is made. The output that was observed after performing tests on a number of persons quite resembled the output of a commercial PCG machine (see figure 2). The observations carried on patients gave quiet satisfactory results sufficient to conclude the condition of the heart in a deterministic way. However, it may require a little skill to observe and analyze the waveform generated by this equipment since it seems to be a little mutilated (see figure 3) which need careful examination.

\section{Conclusion}

The main advantages of this device are - this is an innovative, versatile, cost effective device. The only shortcoming is that certain amount of noise is present in the system that can be seen reflecting in the output. However, this does not interfere with the diagnosis procedures or the inference. The display of signals is a little mutilated that need a careful examination.

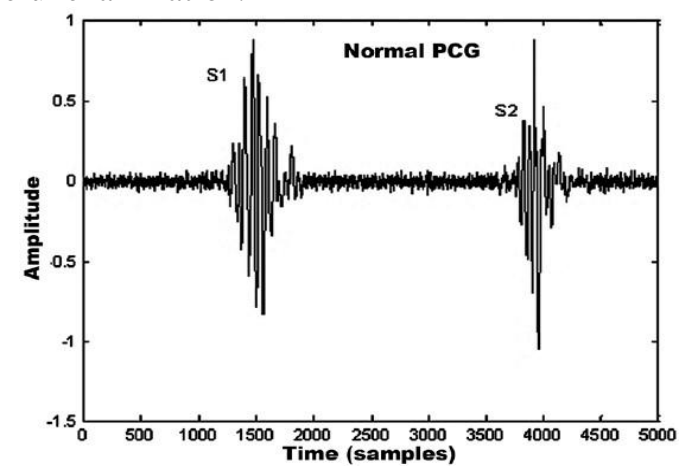

Figure 2: Output of the PCG machine in a digital storage oscilloscope 


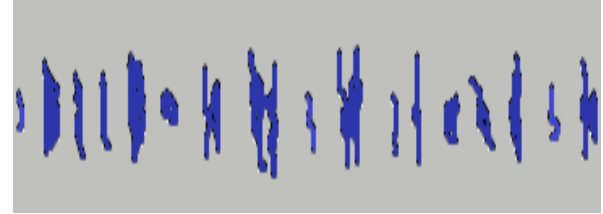

Figure 3: Output of PCG equipment viewed on computer using Sound Wave Editor Software

Since biomedical signals are very noisy and varies considerably from patient to patient, and also depends upon environmental conditions, it is pertinent to consider a time sequence of patient data (i.e., historical data) rather than relying on data captured at a particular instant of time (i.e., instantaneous data).

For more sophisticated analysis a self inferential software can be created that carries sampling, quantification, and encoding of data, and works in conjunction with a linked cardiac database.

\section{References}

[1] A. Mahabuba, J. Vijay Ramnath and G. Anil, Analysis of heart sounds and cardiac murmurs for detecting cardiac disorders using phonocardiography, Journal of Instrumentation Society of India, Vol. 39 No. 1, March 2009.

[2] Shivajirao M. Jadhav, Sanjay L. Nalbalwar \& Ashok A. Ghatol, Modula Neural Network based Arrhythmia Classification System using ECG Signal Data., International Journal of Information Technology and Knowledge Management, January - June 2011, Vol. 4, pp. $205-209$.

[3] Gokhan Bilgin and Oguz Altun, Cardiac Problem Diagnosis with Satistical Neural Networks and Performancee Evaluation by ROC (Receiver Operation Characteristics), Yildiz Technical University, Istanbul.

[4] Curt G. DeGroff, Sanjay Bhatikar, Jean Hertzberg, Robin Shandas, Lilliam Valdes-Crutz, and Roop L. Mahajan, Artificial Neural Network-Based Method of Screening Heart Murmurs in Children, Circulation, Jounal of American Heart Association, Circulation $2001 ; 103 ; 2711-2716$.

[5] Shaikh Abdul Hannan, R. R. Manza, and R. J. Ramteke, Generalized Regression Neural Network and Radial Basis Function for Heart Disease Diagnosis, International Journal of Computer Applications (0975-8887), Volume 7 - No. 13, October 2010.

[6] Sepideh Babei and Amir Garanmayeh, Heart Sound Reproduction based on Neural Network classification of Cardiac Valve disorders using wavelet Transformation of PCG Signals, Computers in Biology and Medicine 39 (2009) 8-15, Elsevier.

[7] Ganmin Ning, Jie Su, yingqi Li, Xiaoying Wang, Chenghong Li, Weimin Yan, amd Xioxinang Zheng, Artificial Neural Network based Model for Cardiovascular Risk Stratification in Hypertension, Medical Biological Engineering Computation (2006) 44; 202208, DIO 10, 1007/s11517-006-0028-2.

[8] Christer Ahlstorm, Processing of the Phonocardiographic signal - method for the intelligent stethoscope. Linkoping University, Institute of Technology, Thesis No: 1253, LIU-TEK-LIC: 2006: 34.

[9] Durand, L.G., de Guise, J., and Guardo, R.A.L.: A microcomputer-based spectrum analyser for phnocardiography. Proceedings of the 32nd Ani.ual Conference on Engineering in Medicine and Biology, vol. 21, p. 2, October 1979.

[10] Kingsley, B.: Acoustic Evaluation of Prosthetic Cardiac Valve in the Audio Spectrum. Journal of Audio Engineering Society Vol. 20, No. 9, pp. 750-755, November 1972. 